\title{
Computer Algebra with SymbolicC++
}


This page intentionally left blank 


\section{Yorick Hardy}

University of Johannesburg

South Africa

\section{Kiat Shi Tan}

Ilog Co., Ltd., Singapore

\section{Willi-Hans Steeb}

University of Johannesburg

South Africa

\section{Computer Algebra with SymbolicC++}

\section{Wo world Scientific}

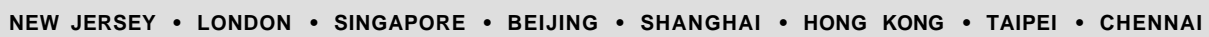




\section{Published by}

World Scientific Publishing Co. Pte. Ltd.

5 Toh Tuck Link, Singapore 596224

USA office: 27 Warren Street, Suite 401-402, Hackensack, NJ 07601

UK office: 57 Shelton Street, Covent Garden, London WC2H 9HE

\section{British Library Cataloguing-in-Publication Data}

A catalogue record for this book is available from the British Library.

\section{COMPUTER ALGEBRA WITH SYMBOLICC++}

Copyright @ 2008 by World Scientific Publishing Co. Pte. Ltd.

All rights reserved. This book, or parts thereof, may not be reproduced in any form or by any means, electronic or mechanical, including photocopying, recording or any information storage and retrieval system now known or to be invented, without written permission from the Publisher.

For photocopying of material in this volume, please pay a copying fee through the Copyright Clearance Center, Inc., 222 Rosewood Drive, Danvers, MA 01923, USA. In this case permission to photocopy is not required from the publisher.

ISBN-13 978-981-283-360-0

ISBN-10 981-283-360-9

ISBN-13 978-981-283-361-7 (pbk)

ISBN-10 981-283-361-7 (pbk) 


\section{Preface}

In this text we show how object-oriented programming can be used to implement a symbolic algebra system and how the system is applied to different areas in mathematics and physics.

In the most restrictive sense, computer algebra is used for the manipulation of scientific and engineering formulae. Usually, a mathematical formula described in the programming languages such as $\mathrm{C}, \mathrm{C}++$ and Java can only be evaluated numerically, by assigning the respective values to each variable. However, the same formula may be treated as a mathematical object in a symbolic algebra system, which allows formal transformation, such as differentiation, integration and series expansion, in addition to the numerical manipulations. This is therefore an indispensable tool for research and scientific computation.

Object-oriented programming has created a new era for programming in computer science as it has been suggested as a possible solution to software development. Basically, object-oriented programming is an important approach to analyzing problems, designing systems and building solutions. By applying this method effectively, the software products become less error prone, easier to maintain, more reusable and extensible.

The purpose of this book is to demonstrate how the features of object-oriented programming may be applied to the development of a computer algebra system. Among the many object-oriented programming languages available nowadays, we have selected $\mathrm{C}++$ as our programming language. It is the most widely used objectoriented programming language, which has been successfully utilized by many programmers in various application areas. The design is based partly on acknowledged principles and partly on solid experience and feedback from actual use. Many experienced individuals and organizations in the industry and academia use $\mathrm{C}++$. In addition to the reasons stated above, we have selected $\mathrm{C}++$ over other objectoriented languages because of its efficiency in execution speed and its utilization of pointers and templates. The Standard Template Library provided by $\mathrm{C}++$ is very helpful for the implementation of a computer algebra system.

Chapter 1 introduces the general notion of Computer Algebra. We discuss the essential properties and requirements of a computer algebra system. Some pitfalls 
and limitations are also listed for reference. Finally, we present a computer algebra system - SymbolicC++. This new system has many advantages over existing computer algebra systems.

Chapter 2 presents the general mathematics for a computer algebra system. We describe how fundamental mathematical quantities are built up to form more complex mathematical structures.

Chapter 3 gives a brief introduction to some computer algebra systems available in the market place, such as Reduce, Maple, Axiom, Mathematica, MuPAD and Maxima. The basic operations are described for each system. Examples are used to demonstrate the features of these systems.

In Chapter 4, we introduce the language tools in $\mathrm{C}++$ such as the this pointer, classes, constructors, and templates. We describe error handling techniques and introduce the concept of exception handling. Examples are also given for demonstration purposes. We also describe recursion. A number of programs illustrate the concepts.

String classes are discussed in Chapter 5. We construct the String data type, which serves as a vehicle for introducing the facilities available in $\mathrm{C}++$. The builtin string class of $\mathrm{C}++$ is also described in detail. A number of examples show the use of this class. This string class of $\mathrm{C}++$ will be used in Symbolic $\mathbf{C}++$.

The Standard Template Library (STL) is introduced in Chapter 6 together with a large number of examples. At the core of the Standard Template Library are the three foundational items: containers, algorithm, and iterators. These items work in conjunction with one another. The built-in class in $\mathrm{C}++$ to deal with complex numbers is also introduced. The built-in classes list, vector, map, complex will be used in SymbolicC++.

Chapter 7 gives a collection of useful classes for computer algebra. We investigate very long integers, rational numbers, quaternions, exact derivatives, vectors, matrices, arrays, bit vectors, finite sets and polynomials. They are the building blocks of mathematics as described in Chapter 2. The internal structures and external interfaces of these classes are described in great detail.

In Chapter 8, we describe how a mathematical expression can be constructed using object-oriented techniques. The computer algebra system SymbolicC++ is introduced and its internal representations and public interfaces are described. Several examples are also presented to demonstrate the functionalities of the system. A symbolic numeric interface is also described.

In Chapter 9, we apply the classes developed in Chapters 7 and 8 to problems in mathematics and physics. Applications are categorized according to classes. Several classes may be used simultaneously to solve a particular problem. Many interest- 
ing problems are presented, such as ghost solutions, Padé approximant, Lie series techniques, Picard's method, Mandelbrot set, etc.

In Chapter 10, we discuss how the programming language Lisp can be used to implement a computer algebra system. We implement an algebraic simplification and differentiation program.

We develop a Lisp system using the object-oriented language $\mathrm{C}++$ in Chapter 11 . $\lambda$-calculus and its implementation in $\mathrm{C}++$ are also be introduced.

Gene expression programming and its use for numerical and symbolic manipulations is studied in Chapter 12. A number of programs are given to illustrate the technique.

The header files of the classes (abstract data type) introduced in Chapters 8 and 9 are listed in Chapter 13.

The level of presentation is such that one can study the subject early on in one's education in science. There is a balance between practical programming and the underlying language. The book is ideally suited for use in lectures on symbolic computation and object-oriented programming. The beginner will also benefit from the book.

The reference list gives a collection of textbooks useful in the study of the computer language $\mathrm{C}++[9],[16],[28],[34],[39],[47],[59]$. For data structures we refer to Budd (1994) [11]. For applications in science we refer to Steeb et al. (1993) [49], Steeb (1994) [50], Steeb (2005) [54] and Steeb et al. (2004) [55].

The $\mathrm{C}++$ programs have been tested with all newer $\mathrm{C}++$ compilers which comply with the $\mathrm{C}++$ Standard and include an implementation of the Standard Template Library.

All programs and header files of SymbolicC++ fall under the GNU General Public License. We omit the following comment (or its equivalent) in all header file and program file listings in the interest of brevity:

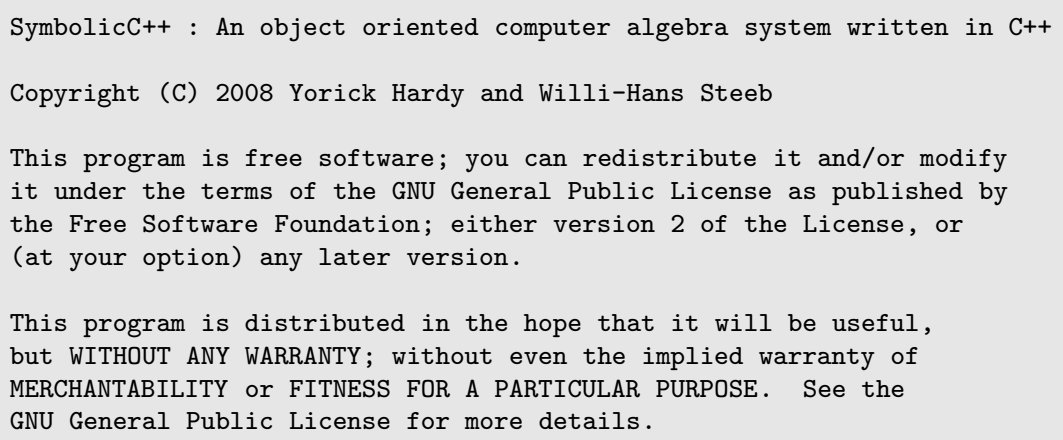


You should have received a copy of the GNU General Public License along with this program; if not, write to the Free Software Foundation, Inc., 51 Franklin Street, Fifth Floor, Boston, MA 02110-1301 USA.

$* /$

The license is included with SymbolicC++ which is available from the web site of the International School for Scientific Computing as described below.

Without doubt, this book can be extended. If you have comments or suggestions, we would be pleased to have them. The email addresses of the authors are:

Yorick Hardy: $\quad$ yhardy@uj.ac.za

yorickhardy@gmail.com

Willi-Hans Steeb: whsteeb@uj.ac.za

steebwilli@gmail.com

SymbolicC++ was developed by the International School for Scientific Computing. The web pages of the International School for Scientific Computing are

http://issc.uj.ac.za/

The web page also provides the header files for Symbolic $\mathbf{C}++$.

Johannesburg, Singapore,

Yorick Hardy

March 2008

Kiat Shi Tan

Willi-Hans Steeb 


\section{Contents}

Preface $\quad$ v

1 Introduction $\quad \mathbf{1}$

1.1 What is Computer Algebra? . . . . . . . . . . . . . 1

1.2 Properties of Computer Algebra Systems . . . . . . . . . . . . . 2

1.3 Pitfalls in Computer Algebra Systems . . . . . . . . . . . . . . . . 3

1.4 Design of a Computer Algebra System . . . . . . . . . . . . 5

2 Mathematics for Computer Algebra 7

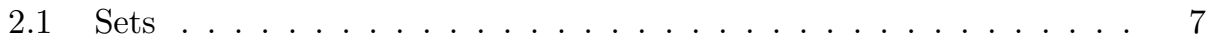

2.2 Rings and Fields . . . . . . . . . . . . . . . . . . . . . . 9

2.3 Integers . . . . . . . . . . . . . . . . . . . . . . . 12

2.4 Rational Numbers ... . . . . . . . . . . . . . . . . 17

2.5 Real Numbers . . . . . . . . . . . . . . . . . . . 20

2.6 Complex Numbers . . . . . . . . . . . . . . . . . 23

2.7 Vectors and Matrices . . . . . . . . . . . . . . . . . . 25

2.8 Determinants . . . . . . . . . . . . . . . . . . . . . . . . . . . . . . 30

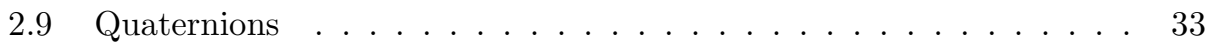

2.10 Polynomials . . . . . . . . . . . . . . . . . . . . . . . . . . . . . . . . . . . . . . . . .

2.11 Gröbner Bases . . . . . . . . . . . . . . . . . . . . . . . . . . . . . . . . .

2.12 Differentiation . . . . . . . . . . . . . . . . . . . . . 52

2.13 Integration . . . . . . . . . . . . . . . . . . . . . . . . . . . . . . . . 53

2.14 Risch Algorithm . . . . . . . . . . . . . . . . . . . . 58

2.15 Commutativity and Non-Commutativity . . . . . . . . . . . 63

2.16 Tensor and Kronecker Product . . . . . . . . . . . . . . . . . 63

2.17 Exterior Product . . . . . . . . . . . . . . 66

3 Computer Algebra Systems $\quad 69$

3.1 Introduction . . . . . . . . . . . . . . . . . 69

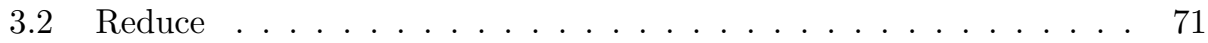

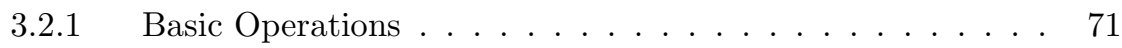

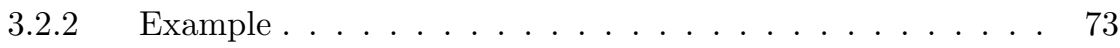

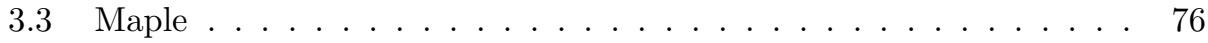

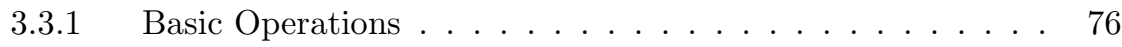

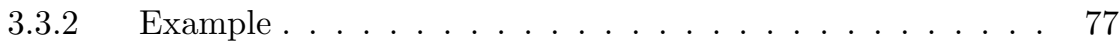

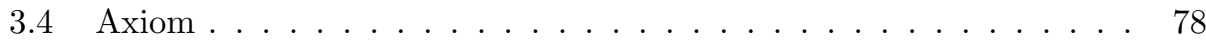


3.4.1 Basic Operations ................. . . 78

3.4.2 Example............................ 80

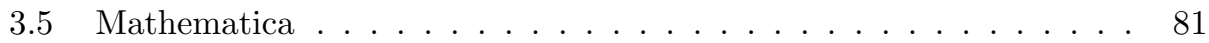

3.5.1 Basic Operations ................. 81

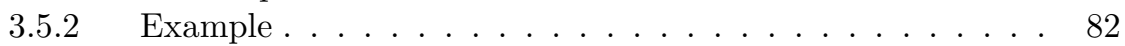

$3.6 \mathrm{MuPAD} \ldots \ldots \ldots \ldots \ldots 2$

3.6.1 Basic Operations . . . . . . . . . . . . . . 82

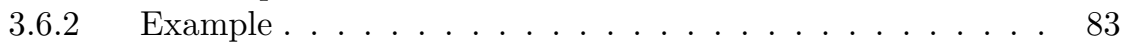

3.7 Maxima ................................ 84

3.7.1 Basic Operations . . . . . . . . . . . . . 84

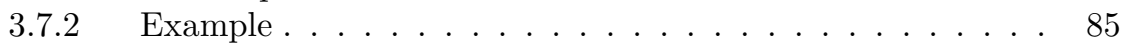

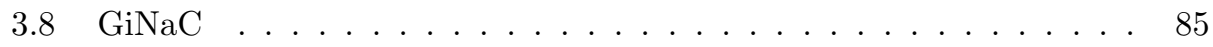

3.8.1 Basic Operations .......................... 85

3.8.2 Example................... 86

4 Tools in $\mathrm{C}++\quad \mathbf{8 7}$

4.1 Pointers and References . . . . . . . . . . . . . . . . 88

4.2 this Pointer . . . . . . . . . . . . . . . . . . . . . . . . 92

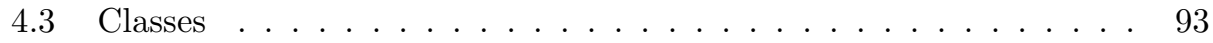

4.4 Constructors and Destructor . . . . . . . . . . . . . . . 96

4.5 Copy Constructor and Assignment Operator . . . . . . . . . . . . 97

4.6 Type Conversion . . . . . . . . . . . . . . . . . . . 98

4.7 Operator Overloading . . . . . . . . . . . . . . . . . . . . . 99

4.8 Class Templates . . . . . . . . . . . . . . . . . . 107

4.9 Function Templates . . . . . . . . . . . . . . . . 110

4.10 Friendship . . . . . . . . . . . . . . . . . . . . 112

4.11 Inheritance . . . . . . . . . . . . . . . . . . . . 113

4.12 Virtual Functions . . . . . . . . . . . . . . . . 116

4.13 Wrapper Class . . . . . . . . . . . . . . . . . 118

4.14 Recursion . . . . . . . . . . . . . . . . . . . 119

4.15 Error Handling Techniques . . . . . . . . . . . . . . . . . . 127

4.16 Exception Handling . . . . . . . . . . . . . . . . . . . . . . . 127

4.17 Run-Time Type Identification . . . . . . . . . . . . . . . . . . . 129

5 String Class 131

5.1 Introduction . . . . . . . . . . . . . . . . . . 131

5.2 A String Class . . . . . . . . . . . . . . . . . . . . . . . . . . . . . . . . . 132

$5.3 \mathrm{C}++$ String Class . . . . . . . . . . . . . . . . 136

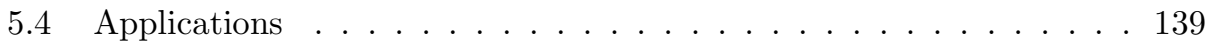

6 Standard Template Library $\quad 145$

6.1 Introduction . . . . . . . . . . . . . . . . . . . . . . . . . . . . . . . 145

6.2 Namespace Concept . . . . . . . . . . . . . . . . . . . . 147

6.3 Vector Class . . . . . . . . . . . . . . . . . . 147

6.4 List Class . . . . . . . . . . . . . . . . . . . . . . 151

6.5 Stack Class . . . . . . . . . . . . . . . . . . . 153

6.6 Queue Class . . . . . . . . . . . . . . . 156 
6.7 Deque Class . . . . . . . . . . . . . . . . . . . . . . 158

6.8 Bitset Class . . . . . . . . . . . . . . . . . . . . . . . . . 159

6.9 Set Class . . . . . . . . . . . . . . . . . . . . . . 161

6.10 Pair Class . . . . . . . . . . . . . . . . . . . . . . . . 163

6.11 Map Class . . . . . . . . . . . . . . . . . . . . . . . 164

6.12 Algorithm Class . . . . . . . . . . . . . . . . . . . . . . 167

6.13 Complex Class . . . . . . . . . . . . . . . . . . . . 169

7 Classes for Computer Algebra $\quad 171$

7.1 Identity Elements . . . . . . . . . . . . . . . . . . . . . . 171

7.2 Verylong Integer Class . . . . . . . . . . . . . . . . . . . 172

7.2 .1 Abstraction . . . . . . . . . . . . . . . . 172

$7.2 .2 \quad$ Data Fields . . . . . . . . . . . . . . . . . . . . . . 174

7.2 .3 Constructors . . . . . . . . . . . . . . . . . . 174

7.2 .4 Operators . . . . . . . . . . . . . . . . . 175

7.2 .5 Type Conversion Operators . . . . . . . . . . . . . . 177

7.2 .6 Private Member Functions . . . . . . . . . . . . . . . 178

7.2 .7 Other Functions . . . . . . . . . . . . . . . . . . 178

7.2 .8 Streams . . . . . . . . . . . . . . . . . . . . . . . 180

7.2 .9 BigInteger Class in Java . . . . . . . . . . . . . . . . . 181

7.3 Rational Number Class . . . . . . . . . . . . . . . . . . . . 181

7.3.1 Abstraction . . . . . . . . . . . . . . . . . 181

$7.3 .2 \quad$ Template Class . . . . . . . . . . . . . . . . . . . . 182

7.3 .3 Data Fields . . . . . . . . . . . . . . . . . . . . . . 182

7.3 .4 Constructors . . . . . . . . . . . . . . . . . . . 182

7.3 .5 Operators . . . . . . . . . . . . . . . . . 183

7.3.6 Type Conversion Operators . . . . . . . . . . . . . . . 184

7.3.7 Private Member Functions . . . . . . . . . . . . . . . . 185

7.3 .8 Other Functions . . . . . . . . . . . . . . . . . . . . 185

7.3 .9 Streams . . . . . . . . . . . . . . . . . 186

7.3.10 Rational Class for Java . . . . . . . . . . . . . . . 186

7.4 Quaternion Class . . . . . . . . . . . . . . . . . 190

$7.4 .1 \quad$ Abstraction . . . . . . . . . . . . . . . . . 190

7.4.2 Template Class . . . . . . . . . . . . . . . . . . . . . 190

7.4 .3 Data Fields . . . . . . . . . . . . . . . . . . . . . . 190

7.4 .4 Constructors . . . . . . . . . . . . . . . . . . 191

7.4 .5 Operators . . . . . . . . . . . . . . . . . 191

7.4 .6 Other Functions . . . . . . . . . . . . . . . . . . . . . . 191

7.4 .7 Streams . . . . . . . . . . . . . . . . . . 192

7.5 Derive Class . . . . . . . . . . . . . . . . . . . . . 192

7.5.1 Abstraction . . . . . . . . . . . . . . . . . . 192

7.5 .2 Data Fields . . . . . . . . . . . . . . . . . . . 193

7.5 .3 Constructors . . . . . . . . . . . . . . . . . 193

7.5 .4 Operators . . . . . . . . . . . . . . . . . . . 193

7.5.5 Member Functions . . . . . . . . . . . . . . . . 193

7.5.6 Possible Improvements . . . . . . . . . . . . . . . . . . . . . 194 
$7.6 \quad$ Vector Class . . . . . . . . . . . . . . . . . . . . . . . . . 195

7.6.1 Abstraction . . . . . . . . . . . . . . 195

7.6 .2 Templates . . . . . . . . . . . . . . . . 196

7.6 .3 Data Fields . . . . . . . . . . . . . . . . . . . 196

7.6 .4 Constructors . . . . . . . . . . . . . . . 196

7.6 .5 Operators . . . . . . . . . . . . . . . 197

7.6.6 Member Functions and Norms . . . . . . . . . . . . . 199

7.6 .7 Streams . . . . . . . . . . . . . . . 200

7.7 Matrix Class . . . . . . . . . . . . . . . . . . 200

7.7.1 Abstraction . . . . . . . . . . . . . . 200

7.7 .2 Data Fields . . . . . . . . . . . . . . . . . 202

7.7 .3 Constructors . . . . . . . . . . . . . . . . . . 202

7.7 .4 Operators . . . . . . . . . . . . . . . 203

7.7.5 Member Functions and Norms . . . . . . . . . . . . 204

7.7.6 Matrix Class for Java . . . . . . . . . . . . . . . . . 208

7.8 Array Class . . . . . . . . . . . . . . . . . . . . . 210

7.8.1 Abstraction . . . . . . . . . . . . . 210

7.8 .2 Data Fields . . . . . . . . . . . . . . . . . . . 211

7.8 .3 Constructors . . . . . . . . . . . . . . . 212

7.8 .4 Operators . . . . . . . . . . . . . . . . . 212

7.8.5 Member Functions . . . . . . . . . . . . . . . . . 214

7.9 Polynomial Class . . . . . . . . . . . . . . . . . . . . . . 215

7.9.1 Abstraction . . . . . . . . . . . . . 215

7.9 .2 Template Class . . . . . . . . . . . . . . . . . 216

7.9 .3 Data Fields . . . . . . . . . . . . . . . . . . . 216

7.9 .4 Constructors . . . . . . . . . . . . . 216

7.9 .5 Operators . . . . . . . . . . . . . . . 217

7.9.6 Type Conversion Operators . . . . . . . . . . . . . . . . 217

7.9.7 Private Member Functions . . . . . . . . . . . . . . 217

7.9 .8 Other Functions . . . . . . . . . . . . . . . . . . 217

7.9 .9 Streams . . . . . . . . . . . . . . . . . . . . . 217

7.9 .10 Example . . . . . . . . . . . . . . . . . . . 218

7.10 Multinomial Class . . . . . . . . . . . . . . . . . . . . . . . 219

7.10 .1 Abstraction . . . . . . . . . . . . . . . 220

7.10 .2 Template Class . . . . . . . . . . . . . . . . . . 220

7.10 .3 Data Fields . . . . . . . . . . . . . . . . . . . 220

7.10 .4 Constructors . . . . . . . . . . . . . . . . . 221

7.10 .5 Operators . . . . . . . . . . . . . . . 221

7.10 .6 Private Member Functions _ . . . . . . . . . . . . . 221

7.10 .7 Other Functions . . . . . . . . . . . . . . . . . . 221

7.10 .8 Streams . . . . . . . . . . . . . . . . . . . 222

7.10 .9 Example. . . . . . . . . . . . . . . . . . . . 222

8 Symbolic Class 223

8.1 Main Header File. . . . . . . . . . . . . . . . . . . . . . . . . . 224

8.2 Memory Management . . . . . . . . . . . . . . . . 225 
8.3 Object-Oriented Design . . . . . . . . . . . . . . . 227

8.3.1 The Expression Tree . . . . . . . . . . . . . . 227

8.3.2 Polymorphism of the Expression Tree . . . . . . . . . . . . 228

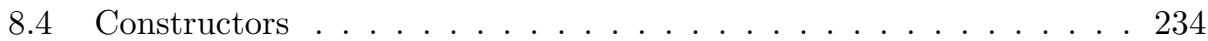

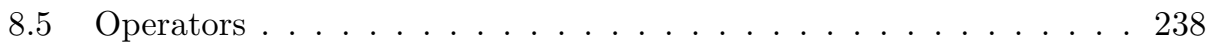

8.6 Functions and Member Functions . . . . . . . . . . . . . . 239

8.6.1 Functions . . . . . . . . . . . . . . . 252

8.7 Simplification . . . . . . . . . . . . . . . . . 253

8.7.1 Canonical Forms . . . . . . . . . . . . . . . . . . . 254

8.7.2 Simplification Rules and Member Functions . . . . . . . . . 254

8.8 Commutativity . . . . . . . . . . . . . . . . 256

8.9 Symbolic and Numeric Interface . . . . . . . . . . . . . . . . 258

8.10 Example Computation . . . . . . . . . . . . . . . . . . 259

9 Applications 263

9.1 Bitset Class . . . . . . . . . . . . . . . . . . . . . 264

9.2 Verylong Class . . . . . . . . . . . . . . . . . 267

9.2.1 Big Prime Numbers . . . . . . . . . . . . . . 267

9.2.2 Gödel Numbering . . . . . . . . . . . . . . . . 270

9.2.3 Inverse Map and Denumerable Set . . . . . . . . . . . . . 275

9.3 Verylong and Rational Classes . . . . . . . . . . . . . . . . 277

9.3.1 Logistic Map . . . . . . . . . . . . . . . . . . . . . 277

9.3.2 Contracting Mapping Theorem . . . . . . . . . . . . 278

9.3.3 Ghost Solutions . . . . . . . . . . . . . . . . 281

9.3.4 Iterated Function Systems . . . . . . . . . . . . . . 282

9.4 Verylong, Rational and Derive Classes . . . . . . . . . . . . . . 285

9.4.1 Logistic Map and Ljapunov Exponent . . . . . . . . . . . . 285

9.5 Verylong, Rational and Complex Classes . . . . . . . . . . . . . 287

9.5.1 Mandelbrot Set . . . . . . . . . . . . . . . . 287

9.6 Symbolic Class . . . . . . . . . . . . . . . . . . . . . 288

9.6.1 Polynomials . . . . . . . . . . . . . . . 288

9.6.2 Cumulant Expansion . . . . . . . . . . . . . . . 299

9.6.3 Exterior Product . . . . . . . . . . . . . . . 301

9.7 Symbolic Class and Symbolic Differentiation . . . . . . . . . . . . 302

9.7.1 First Integrals . . . . . . . . . . . . . . . . . . 302

9.7 .2 Spherical Harmonics . . . . . . . . . . . . . . . . . . 304

9.7.3 Nambu Mechanics . . . . . . . . . . . . . . . 306

9.7.4 Taylor Expansion of Differential Equations . . . . . . . . . 307

9.7.5 Commutator of Two Vector Fields . . . . . . . . . . . . . . 311

9.7.6 Lie Derivative and Killing Vector Field . . . . . . . . . . . . 312

9.8 Matrix Class . . . . . . . . . . . . . . . . . . . . . . 314

9.8.1 Hilbert-Schmidt Norm . . . . . . . . . . . . . . . . . . 314

9.8.2 Lax Pair and Hamilton System . . . . . . . . . . . . . . 315

9.8.3 Padé Approximant . . . . . . . . . . . . . . . . . . 317

9.9 Array and Symbolic Classes . . . . . . . . . . . . . . . . 320

9.9.1 Pseudospherical Surfaces and Soliton Equations . . . . . . . 320 
9.10 Polynomial and Symbolic Classes . . . . . . . . . . . . . . . . . 321

9.10.1 Picard's Method . . . . . . . . . . . . . . . 321

9.11 Lie Series Techniques . . . . . . . . . . . . . . . . . . . 323

9.12 Spectra of Small Spin Clusters . . . . . . . . . . . . . . . . . 326

9.13 Nonlinear Maps and Chaotic Behavior . . . . . . . . . . . . . . . . 328

9.14 Numerical-Symbolic Application . . . . . . . . . . . . . . . . 330

9.15 Bose Systems . . . . . . . . . . . . . . . . . . . . . . . . . . . 332

9.16 Grassman Product and Lagrange Multipliers . . . . . . . . . . . . . 333

9.17 Interpreter for Symbolic Computation . . . . . . . . . . . . . . 334

10 LISP and Computer Algebra 341

10.1 Introduction . . . . . . . . . . . . . . . . . . . 341

10.2 Basic Functions of LISP . . . . . . . . . . . . . . . . . . . . . . . . . . . . . . . . . . . . . . . . . . . . .

10.3 LISP Macros and Infix Notation . . . . . . . . . . . . . . . . . 349

10.4 Examples from Symbolic Computation . . . . . . . . . . . . . . . 351

10.4.1 Polynomials . . . . . . . . . . . . . . . 351

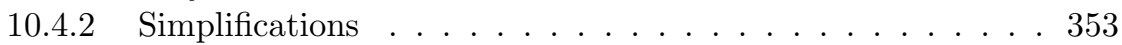

10.4.3 Differentiation ................. . . 362

10.5 LISP, Haskell and Computer Algebra . . . . . . . . . . . . . . . . . 365

10.5.1 A simple computer algebra system in LISP . . . . . . . . . 365

10.5.2 A simple computer algebra system in Haskell . . . . . . . . 367

11 Lisp using $\mathrm{C}++\quad 371$

11.1 Lisp Operations in $\mathrm{C}++\ldots \ldots$. . . . . . . . . . . . . . . . . . . . 371

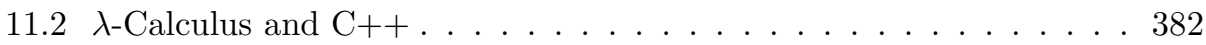

11.2.1 $\lambda$-Calculus . . . . . . . . . . . . . . . 382

11.2.2 C++ Implementation . . . . . . . . . . . . 385

12 Gene Expression Programming 401

12.1 Introduction . . . . . . . . . . . . . . . . . . . . 401

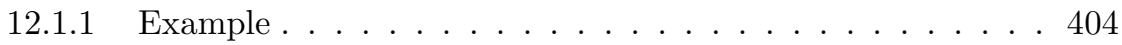

12.2 Multi Expression Programming . . . . . . . . . . . . . . 416

13 Program Listing $\quad 423$

13.1 Identities . . . . . . . . . . . . . . . . . . . . . . . . . . . . . . . . . . . . . . . . . . .

13.2 Verylong Class . . . . . . . . . . . . . . . . . . . . . . . . . . . . . . . . . . . . . 424

13.3 Rational Class . . . . . . . . . . . . . . . . . . . . . . . . . . . . . . . . . . . . . . . .

13.4 Quaternion Class . . . . . . . . . . . . . . . . . . . . . . . . . . . . . . . . . . . . . . 445

13.5 Derive Class . . . . . . . . . . . . . . . . . . . . . . . . . . . . . . . . . . . . . . . . . . . . . . .

13.6 Vector Class . . . . . . . . . . . . . . . . . . . . . . . . . . . . . . . . . . . . . . . . . . . . . .

13.6.1 Vector Class . . . . . . . . . . . . . . . . . . . 449

13.6.2 Vector Norms . . . . . . . . . . . . . . . . . . 454

13.7 Matrix Class . . . . . . . . . . . . . . . . . . . . . . . . . . . . . . . . . . . . . . . . . . . .

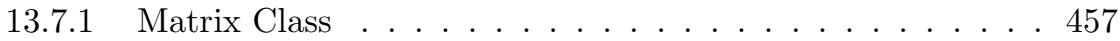

13.7.2 Matrix Norms . . . . . . . . . . . . . . . 466

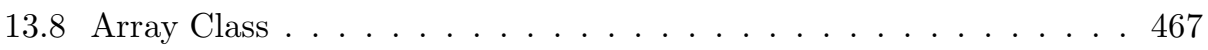

13.9 Polynomial Class . . . . . . . . . . . . . . . . . . . 473 
13.10 Multinomial Class . . . . . . . . . . . . . . . . . . . . 485

13.11 Symbolic Class . . . . . . . . . . . . . . . . . . . 496

13.11.1 Main Header File . . . . . . . . . . . . . . . . . . . . . . . 499

13.11.2 Memory Management . . . . . . . . . . . . . . . . 507

13.11 .3 Constants . . . . . . . . . . . . . . . . . . . 510

13.11 .4 Equations . . . . . . . . . . . . . . . . . . . 510

13.11 .5 Functions . . . . . . . . . . . . . . . . . . 512

13.11 .6 Numbers . . . . . . . . . . . . . . . . . . . . 525

13.11 .7 Products . . . . . . . . . . . . . . . 536

13.11 .8 Sums . . . . . . . . . . . . . . . . . 545

13.11 .9 Symbols . . . . . . . . . . . . . . . . . . . . 5 551

13.11.10 Symbolic Expressions _ . . . . . . . . . . . . . 555

13.11.11 Symbolic Matrices . . . . . . . . . . . . . . . . 569

13.11 .12 Errors . . . . . . . . . . . . . . . . . . . 572

$\begin{array}{ll}\text { Bibliography } & 575\end{array}$

Index $\quad 579$ 